\title{
Sizes of Confirmed Globular Clusters in NGC 5128: A Wide-Field High-Resolution Study ${ }^{1}$
}

\author{
Matías Gómez \\ Grupo de Astronomía, Depto. de Física, Universidad de Concepción, Casilla 160-C, \\ Concepción, Chile \\ matias@astro-udec.cl \\ Kristin A. Woodley \\ Department of Physics 83 Astronomy, McMaster University, Hamilton ON L8S 4M1, \\ Canada \\ woodleka@physics.mcmaster.ca
}

\begin{abstract}
Using Magellan/IMACS images covering a 1.2 x 1.2 sq. degree FOV with seeing of 0.4"-0.6", we have applied convolution techniques to analyse the light distribution of 364 confirmed globular cluster in the field of NGC 5128 and to obtain their structural parameters. Combining these parameters with existing Washington photometry from Harris et al. (2004), we are able to examine the size difference between metal-poor (blue) and metal-rich (red) globular clusters. For the first time, this can be addressed on a sample of confirmed clusters that extends to galactocentric distances about 8 times the effective radius, $\mathrm{R}_{e f f}$, of the galaxy. Within $1 \mathrm{R}_{e f f}$, red clusters are about $30 \%$ smaller on average than blue clusters, in agreement with the vast majority of extragalactic globular cluster systems studied. As the galactocentric distance increases, however, this difference becomes negligible. Thus, our results indicate that the difference in the clusters' effective radii, $\mathrm{r}_{e}$, could be explained purely by projection effects, with red clusters being more centrally concentrated than blue ones and an intrinsic $\mathrm{r}_{e}-\mathrm{R}_{g c}$ dependence, like the one observed for the Galaxy.
\end{abstract}

Subject headings: galaxies: elliptical and lenticular, cD - galaxies: individual (NGC 5128) - galaxies: star clusters - globular clusters: general

\footnotetext{
${ }^{1}$ This paper includes data gathered with the 6.5 meter Magellan Telescopes located at Las Campanas Observatory, Chile
} 


\section{Introduction}

Since sizes and structural parameters of globular clusters (GCs) in different GC systems (GCSs) have first been obtained, it has become clear that some of these properties correlate with global properties of their host galaxies (see for example Jordán et al. 2005; Brodie \& Strader 2006). The existence of the so called fundamental plane relation for an increasing number of studied GCSs seems to confirm that GCs populate a narrow region in this parameter space (Djorgovski 1995; McLaughlin 2000; McLaughlin \& van der Marel 2005; Barmby et al. 2007). However, there are puzzling trends that are still awaiting confirmation and need to be addressed using larger samples of GCs.

It is necessary to study structural parameters of GCs and GC-like objects in different environments before definitive statements can be made regarding their formation. Among the structural parameters that can be studied, the effective (or half-light) radius is of particular importance. Models have shown that this quantity remains fairly constant throughout the entire GC lifetime (Spitzer \& Thuan 1972; Aarseth \& Heggie 1998), making it a good indicator of proto-GC sizes that are still observable today. A decade ago, HST observations unveiled a systematic size difference between red and blue GCs (Kundu \& Whitmore 1998). Since then, multiple studies have found that the blue GCs are between 17\% - 30\% larger than their metal-rich counterparts in both spirals and early-type galaxies (Kundu et al. 1999; Puzia et al. 1999; Larsen et al. 2001a,b); Kundu \& Whitmore 2001; Barmby et al. 2002; Jordán et al. 2005). However, most of these studies have made use of HST observations and examine only the innermost regions of the galaxy or small fields in regions at galactocentric distances greater than the galaxy's effective radius.

According to Larsen \& Brodie (2003), the systematic size difference between red and blue GCs is caused merely by a projection effect. Since red (metal-rich) GCs are found to be more centrally concentrated than blue (metal-poor ones) in early type galaxies (Côté et al. 2001; Dirsch et al. 2003; Woodley et al. 2005, among others), the red GCs will appear to lie, on average, at a smaller galactocentric distance. The red clusters will on average be smaller than the blue clusters assuming that both types shares the same relation between the GC size and galactocentric distance. The relation $\mathrm{r} \sim \sqrt{\mathrm{R}_{\mathrm{gc}}}$ was first found in the Milky Way by van den Bergh et al. (1991). In this scenario, the difference between the cluster sizes should be most apparent at small galactocentric distance and should decrease strongly beyond 1 galaxy effective radius (Larsen \& Brodie 2003).

Alternatively, Jordán (2004) suggests that this effect could be explained by an intrinsic difference between metal-rich and metal-poor GCs. Assuming half-mass radii that are independent of metallicity, effects of mass segregation combined with a metallicity-dependent stellar lifetime should lead to different sizes between the blue and red clusters. The brightest 
stars would be more massive and more centrally concentrated for the metal-rich GCs. This scenario should have little to no dependence on a cluster's distance from the center of its parent galaxy.

In a recent study, Spitler et al. (2006) analysed the GCS of NGC 4594 (Sombrero, at a distance of $\sim 9 \mathrm{Mpc}$ ) using a six-image mosaic from HST/ACS. They confirm that within the inner 2 arcmin $\left(2.2 \mathrm{R}_{e f f}\right)$, the metal-rich GCs are, on average, $17 \%$ smaller than the metalpoor clusters. However, the size difference becomes negligible at $\sim 3$ arcmin, corresponding to $\sim 3.4 \mathrm{R}_{e f f}$, where $\mathrm{R}_{\text {eff }}=0.89$ arcmin (Baggett et al. 1998).

To further understand the sizes of red and blue clusters, we need a homogeneous survey of a GCS with the ability to eliminate contaminating sources, high resolution to measure structural parameters, and over a large range in galactocentric distance.

NGC 5128 is the nearest giant elliptical galaxy, at a distance of 3.8 Mpc (McLaughlin et al. 2007). Its GCs are thus easily resolvable with sub-arcsecond seeing (Harris et al. 2006). In this paper we present effective radius results for 337 GCs from the Woodley et al. (2007) catalog that are confirmed GCs by either radial velocity measurement from various studies (see the references in Woodley et al. 2007) or are resolved by HST/ACS images (Harris et al. 2006). We also present the effective radii of 27 GCs newly confirmed through radial velocity measurements using the Baade 6.5-m telescope with the instrument LDSS-2 (data in preparation for publication). This list represents a clean sample of confirmed clusters. All of these also have ellipticities less than 0.4 and effective radii less than 8 pc, both of which are consistent with normal GC properties in NGC 5128. We find that only an additional $2.4 \%$ of GCs from the Woodley et al. (2007) catalog have effective radii greater than the 8 pc boundary we have imposed here (to be discussed in detail in Gómez \& Woodley, 2008, in preparation). Those few GCs are not considered here as our purpose is to establish the effective radius trends within the bulk of the GC population.

\section{Observations}

On the night of April 9, 2006, 25 fields were imaged with the Magellan $6.5 \mathrm{~m}$ telescope using the Inamori Magellan Areal Camera and Spectrograph (IMACS). In the highest imaging resolution, IMACS offers a FOV of 15.4 arcmin on a side, composed of a mosaic of $82 \mathrm{Kx} 4 \mathrm{KCDs}$ with a scale of $0.111 \mathrm{arcsec} /$ pixel. Our observational material will be fully discussed in Harris et al. 2008 (in preparation). The total field of view of our images is roughly $1.2 \times 1.2$ square degrees and the average seeing is about 0.5 " across the entire field with individual frames ranging from 0.35 " to 0.7 ". Images were acquired through B (on 16 
of the 25 fields) and R (on all 25 fields) filters with both 10 second and 300 second exposures to avoid saturation of the brightest clusters.

We have identified all GCs in the catalog of Woodlev et al. (2007) on our IMACS frames2. We have run the code ISHAPE (Larsen 1999, 2001) individually on each GC in our R filtered IMACS frames, using a stellar point spread function (PSF) modelled from the chip in which the cluster is located. For this, typically 20-30 stars were chosen in each frame and measured with standard tools in IRAF 3 . ISHAPE convolves the PSF with analytical profiles and compares the result with the input image until a best match is achieved.

As the analytical model, we chose King (1962) profiles, given their simplicity and because they are known to provide a good fit to a large family of GCs in different environments. Moffat functions were also tried, but they do not improve the fits except for a handful of large and very elliptical sources that we are not considering for the present study. They will be discussed as special cases in a forthcoming paper. Possible systematic effects in the sizes, arising from the choice of a particular model are discussed in Larsen (1999). However, the effective radius seems to be independent of the model for sources that have a similar extension to the stellar PSF, as in our case. For a recent comparison between different models, the reader is referred to Barmby et al. (2007) and McLaughlin et al. (2007).

King (1962) profiles are defined by a core-radius $r_{c}$ and a concentration index, which we define here as $c=\mathrm{r}_{t} / \mathrm{r}_{c}$, where $\mathrm{r}_{t}$ is the tidal radius of the cluster. Usually, the concentration parameter is the most uncertain one to constrain (Larsen 2001), but given the high spatial resolution of our IMACS images, we were able to fit this along with the ellipticity, position angle $(\mathrm{PA})$ and $\mathrm{r}_{c}$.

The sizes quoted by ISHAPE were transformed into effective radii using the approximation $\mathrm{r}_{e} / \mathrm{r}_{c} \approx 0.547 c^{0.486}$, good to $\pm 2 \%$ for $c>4$ (Larsen 2001). The median value for the concentration parameter for GCs in NGC 5128 was $c=39.4 \pm 10.2$. Uncertainties in the effective radius were estimated by the standard deviation of the determined value using King profiles with fixed concentration indices of 15, 30, and 100. These concentration parameters were chosen based on typical values observed in our Galaxy as well as the concentration parameters fit freely with ISHAPE for the NGC 5128 data. The $\mathrm{r}_{e}$ determined for any given cluster with varying concentration parameters does not vary more than $\sim 10 \%$ for the aver-

\footnotetext{
${ }^{2}$ Note that the positions of two GCs have been corrected: GC0001 with a corrected right ascension of $13^{h} 25^{m} 1.16^{s}(\mathrm{~J} 2000)$ and GC0002 with corrected declination of $-43^{\circ} 02^{\prime} 42.9^{\prime \prime}$ (J2000).

${ }^{3}$ IRAF is distributed by the National Optical Astronomy Observatory, which is operated by the Association of Universities for Research in Astronomy Inc., under cooperative agreement with the National Science Foundation.
} 
age GC. The concentration parameter, c, is the most uncertain of the fitted parameters. The extension of the GC is a secondary uncertainty. A GC at the distance of NGC 5128, with an effective radius of $6 \mathrm{pc}$, would span a diameter of $0.6 "$, marginally larger than the typical stellar FWHM. For smaller or more compact objects, the instrisic size can be as small as 0.1 ", i.e. completely blurred even with sub-arcsecond imaging.

\section{Results}

We have 69 GCs in common with the HST/ACS structural parameter study of Harris et al. (2006). Their $\mathrm{r}_{e}$ values, derived through an isophotal analysis of the resolved clusters, and discussed fully in McLaughlin et al. (2007), serve as an external comparison and quality test for our measurements. Figure 1 shows clearly that there is good agreement in the $r_{e}$ values determined by these independent techniques. We have also examined our measured $\mathrm{r}_{e}$ as a function of ellipticity and luminosity and also found no notable correlation.

In an upcoming paper, (Gómez \& Woodley, 2008, in preparation), we will discuss the structural parameters in detail as well as the new GCs discovered with the Baade $6.5 \mathrm{~m}$ telescope (mentioned above) that have been used in this study. Here, we focus on the dependence of the GC sizes as a function of galactocentric radius, $\mathrm{R}_{g c}$ for the GC subpopulations. A metallicity break has been chosen to represent the red or metal-rich $([\mathrm{Fe} / \mathrm{H}]>-1)$ and blue or metal-poor $([\mathrm{Fe} / \mathrm{H}]<-1)$ subpopulations of clusters, following the studies of Larsen \& Brodie (2003); Harris et al. (2004); Woodley et al. (2005); Gómez et al. (2006); Woodlev et al. (2007). The $[\mathrm{Fe} / \mathrm{H}]$ values were obtained from a $C-T_{1}$ transformation (Harris \& Harris 2002) assuming $\mathrm{E}(\mathrm{B}-\mathrm{V})=0.11$ (Schlegel et al. 1998).

Figures 2 and 3 show that, within one effective radius of the galaxy, the red clusters are significantly smaller than the blue ones by $\sim 30 \%$. As galactocentric distance increases, however, this difference tends to disappear and beyond a distance of $12 \mathrm{kpc}$ (corresponding to $\sim 2.3 \mathrm{R}_{\text {eff }}$ ), no difference remains. We have performed a Spearman test to study the trend between metallicity and $\mathrm{r}_{e}$. The Spearman non-parametric rank-correlation coefficient ranges from -1 to 1 , with -1 for a complete anti-correlation, 0 for no correlation and 1 for a complete correlation. We found the coefficients to be -0.4 for $\mathrm{R}_{g c}<1 \mathrm{R}_{e f f}$, -0.1 for 1 $\mathrm{R}_{e f f}<\mathrm{R}_{g c}<2 \mathrm{R}_{e f f}, 0.1$ for $2 R_{e f f}<\mathrm{R}_{g c}<3 \mathrm{R}_{\text {eff }}$, and 0.1 for $\mathrm{R}_{g c}>3 \mathrm{R}_{e f f}$. In addition, the Spearman test gives the probability that the two datasets are uncorrelated as well. The values read $0.01,0.19,0.43$ and 0.34 , respectively, for the four radial bins presented above. Thus, there is a small, but virtually confirmed anti-correlation within $1 \mathrm{R}_{\text {eff }}$.

This size trend is even more clear in Figure 3 . Within $1 \mathrm{R}_{\text {eff }}$ of the galaxy, the metal- 
rich and metal-poor clusters have median effective radii of $1.94 \pm 0.19$ and $2.98 \pm 0.20 \mathrm{pc}$, respectively. However, outside of $1 \mathrm{R}_{e f f}$, the median $\mathrm{r}_{e}$ is identical within uncertainties.

Figure 4 shows $\mathrm{r}_{e}$ as a function of projected $\mathrm{R}_{g c}$ for the GCs in NGC 5128 and in the Milky Way. The metal-poor GCs in NGC 5128 do not follow the $\mathrm{r}_{e}-\mathrm{R}_{g c}$ relationship that is evident in the metal-rich GCs. The Milky Way data, on the other hand, has been shown to host the $\mathrm{r}_{e}-\mathrm{R}_{g c}$ relationship for both metallicity populations, first noted by van den Bergh et al. (1991), using a 3-dimensional $\mathrm{R}_{g c}$. However, in projection, the metalpoor GCs in the Milky Way do not appear vastly different from those in NGC 5128.

\section{Discussion}

The existence of a systematic difference in the effective radii of blue and red clusters has been extensively studied in other galaxies, with blue clusters typically found to be $17-30 \%$ larger than red ones (see Section 1). However, in NGC 5128, Harris et al. (2002) did not find any correlation between color and size for a sample of 27 GCs using HST/WFPC2. In a subsequent study, Gómez et al. (2006) found the red GCs to have larger median sizes compared to the blue clusters for a sample of 38 objects, with Magellan/MagIC. Both studies were based on small field images, involved small sample sizes, and were centered at large $\mathrm{R}_{g c}$. (At the smallest $\mathrm{R}_{g c}$, this was more than 2 times farther than the $\mathrm{R}_{\text {eff }}$ of the galaxy light.) According to Larsen \& Brodie (2003), the average sizes of red and blue clusters should be similar at about $1 \mathrm{R}_{\text {eff }}$ and beyond, if projection effects are to account for the size difference. The results from Harris et al. (2002) and Gómez et al. (2006) are consistent with this scenario, bearing in mind the low number statistics of their studies.

The only two studies thus far with a large enough sample of GCs that also extends beyond $1 \mathrm{R}_{\text {eff }}$, are this work and Spitler et al. (2006). Both show that the red clusters are

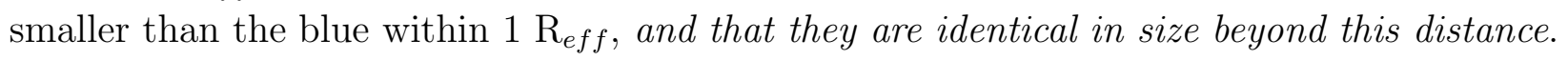
In the ACS Virgo Cluster Survey, Jordán et al. (2005) have studied the sizes of GCs in 67 early-type galaxies. Their analysis reaches about 3 times the effective radius of the massive ellipticals studied and these GCSs dominate their sample. However, their results, favoring red clusters being consistently smaller than blue clusters are dominated by the inner GCs in these galaxies.

Our sample, consists of only confirmed GCs which have been analyzed homogeneously, span a projected galactocentric distance of up to $50 \mathrm{kpc}$, i.e., 8 galaxy $\mathrm{R}_{\text {eff }}$. Thus, we are able to draw conclusions about the origin of the size difference with a sample that is both uncontaminated and much more spatially extended than in previous studies. 
As is evident from Figs. 2, 3] and 4 metal-poor clusters do not show an $\mathrm{r}_{e}-\mathrm{R}_{g c}$ relationship. Jordán et al. (2005) analyse this trend for metal-poor clusters in their samples and conclude that they are too shallow compared to the Galaxy for the projection effects to account for the size difference. Our results for NGC 5128 agree with this, but at the same time make it clear that the metal-poor subpopulation does not represent the global $\mathrm{r}_{e}-\mathrm{R}_{g c}$ trend. In fact, only metal-rich clusters show it. Therefore, projection effects can account for the observed size differences without the need of intrinsic formation and destruction mechanisms between red and blue clusters.

\section{Conclusions}

Using a contaminant-free sample of 364 GCs in NGC 5128, confirmed with radial velocity measurements or by resolved HST images, we have measured effective radii using ISHAPE. Our results indicate that the blue or metal-poor clusters do not show any significant $\mathrm{r}_{e}-\mathrm{R}_{g c}$ relation. However, the red or metal-rich GCs do show a steep relation in which red clusters within $1 \mathrm{R}_{\text {eff }}$ of the galaxy's light are $30 \%$ smaller than the blue clusters. Beyond this distance there is no indication for a size difference between the two metallicity populations. This finding in NGC 5128, not previously seen in any other early-type galaxy, supports the more tentative findings of the Sombrero galaxy's GCS (Spitler et al. 2006). Both studies support the idea that the size differences are most likely caused by projection effects (Larsen \& Brodie 2003) and not by intrinsic physical differences between the two subgroups.

Acknowledgements: M.G. and K.A.W. thank Dean McLaughlin for use of HST structural parameters in advance of publication. M.G. thanks the Dept. of Physics and Astronomy at McMaster University and especially Bill and Gretchen Harris for their hospitality. K.A.W. thanks NSERC and Bill Harris for financial support, and also the Depto. de Física at the Universidad de Concepción, especially Doug Geisler, for their hospitality. We thank the anonymous referee for her/his valuable suggestions and comments. 


\section{REFERENCES}

Aarseth, S. J. \& Heggie, D. C. 1998, MNRAS, 297, 794

Barmby, P., Holland, S., \& Huchra, J. P. 2002, AJ, 123, 1937

Barmby, P., McLaughlin, D. E., Harris, W. E., Harris, G. L. H., \& Forbes, D. A. 2007, AJ, 133,2764

Baggett, W. E., Baggett, S. M., \& Anderson, K. S. 1998, AJ, 116, 1626

Brodie, J. P. \& Strader, J. 2006, ARA\&A, 44, 193

Côté, P., McLaughlin, D. E., Hanes, D. A., Bridges, T. J., Geisler, D., Merritt, D., Hesser, J. E., Harris, G. L. H., \& Lee, M. G. 2001, ApJ, 559, 828

de Vaucouleurs, G. \& Pence, W. D. 1978, AJ, 83, 1163

Dirsch, B., Richtler, T., Geisler, D., Forte, J. C., Bassino, L. P., \& Gieren, W. P. 2003, AJ, 125,1908

Djorgovski, S. 1995, ApJ, 438, 29

Drinkwater, M. J., Jones, J. B., Gregg, M. D., \& Phillipps, S. 2000, PASA, 17, 227

Gómez, M., Geisler, D., Harris, W. E., Richtler, T., Harris, G. L. H., \& Woodley, K. A. 2006, A\&A, 447, 877

Harris, G. L. H., Harris, W. E., \& Geisler, D. 2004, AJ, 128, 723

Harris, W. E. 1996, AJ, 112, 1487

Harris, W. E., \& Harris, G. L. H. 2002, AJ, 123, 3108

Harris, W. E., Harris, G. L. H., Holland, S. T., \& McLaughlin, D. E. 2002, AJ, 124, 1435

Harris, W. E., Harris, G. L. H., Barmby, P., McLaughlin, D. E., \& Forbes, D. A. 2006, AJ, 132,2187

Jordán, A. 2004, ApJ, 613L, 117

Jordán, A., Côté, P., Blakeslee, J. P., Ferrarese, L., McLaughlin, D. E., Mei, S., Peng, E. W., Tonry, J. L., Merritt, D., Milosavljević, M., Sarazin, C. L., Sivakoff, G. R., \& West, M. J. 2005, ApJ, 634, 1002 
King, I. R. 1962, AJ, 67, 471

Kundu, A. \& Whitmore, B. C. 1998, AJ, 116, 2841

Kundu, A., Whitmore, B. C., Sparks, W. B., Macchetto, F. D., Zepf, S. E., \& Ashman, K. M. 1999, ApJ, 513, 733

Kundu, A. \& Whitmore, B. C. 2001, AJ, 121, 2950

Larsen, S. S. 1999, A\&AS, 139, 393

Larsen, S. S. 2001, AJ, 122, 1782

Larsen, S. S., Brodie, J. P., Huchra, J. P., Forbes, D. A., \& Grillmair, C. J. 2001a, AJ, 121, 2974

Larsen, S. S., Forbes, D. A., \& Brodie, J. P. 2001b, MNRAS, 327, 1116

Larsen, S. S. \& Brodie, J. P. 2003, ApJ, 593, 340

McLaughlin, D. E. 2000, ApJ, 539, 618

McLaughlin, D. E. \& van der Marel, R. P. 2005, ApJS, 161, 304

McLaughlin, D. E., Barmby, P., Harris, W. E., Harris, G. L. H., \& Forbes, D. A. 2007, MNRAS, submitted

Puzia, T. H., Kissler-Patig, M., Brodie, J. P., \& Huchra, J. P. 1999, AJ, 118, 2734

Schlegel, D. J., Finkbeiner, D. P., \& Davis, M. 1998, ApJ, 500, 525

Spitler, L. R., Larsen, S. S., Strader, J., Brodie, J. P., Forbes, D. A., \& Beasley, M. A, 2006, AJ, 132, 1593

Spitzer, L. Jr., Thuan, T. X. 1972, ApJ, 175, 31

van den Bergh, S., Morbey, C., \& Pazder, J. 1991, ApJ, 375, 594

Woodley, K. A., Harris, W. E., \& Harris, G. L. H, 2005, AJ, 129, 2654

Woodley, K. A., Harris, W. E., Beasley, M. A., Peng, E. W., Bridges, T. J., Forbes, D. A., \& Harris, G. L. H 2007, AJ, 134, 494

This preprint was prepared with the AAS LATEX macros v5.0. 


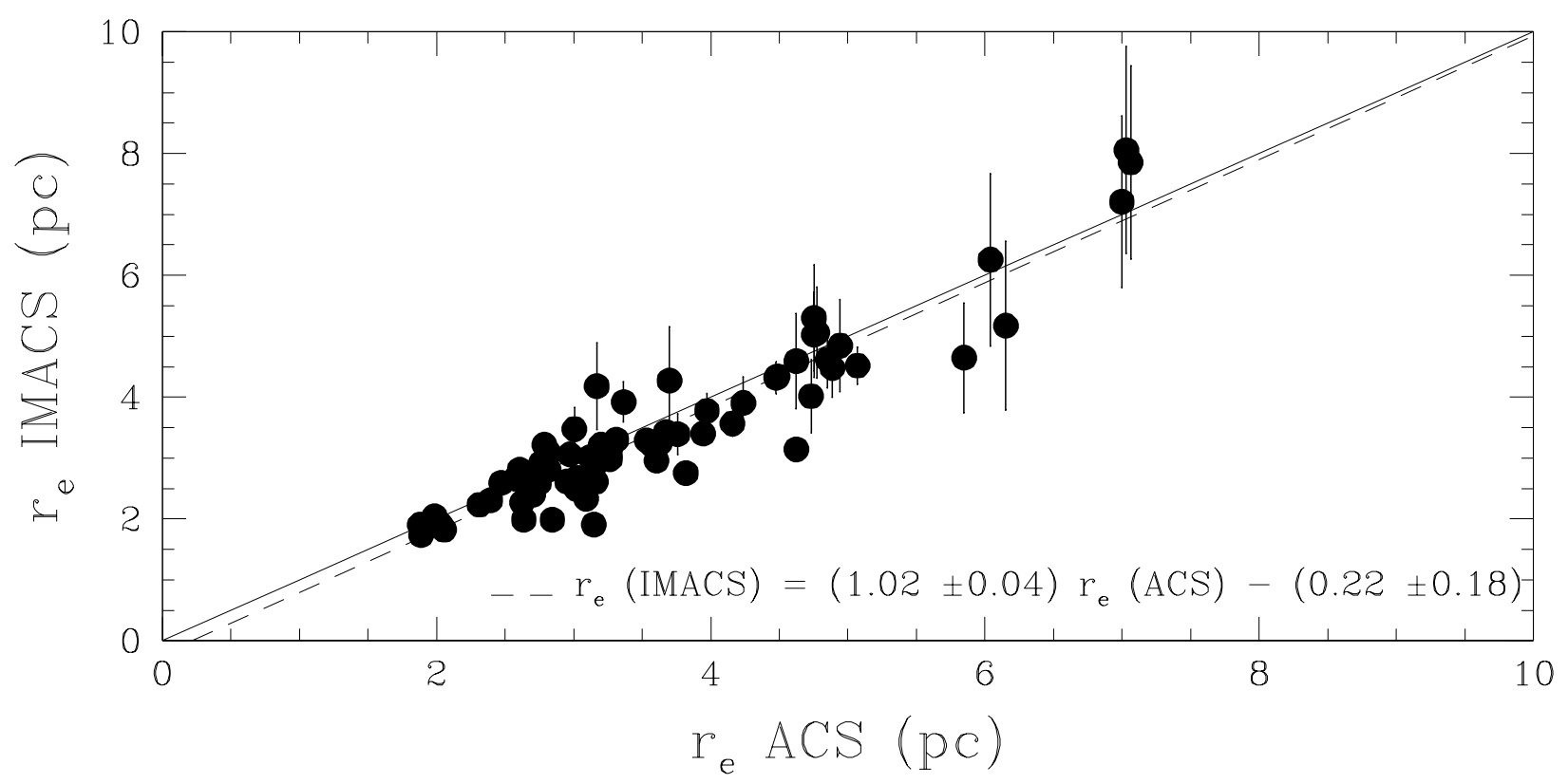

Fig. 1.- Comparison of effective radius $\mathrm{r}_{e}$, in parsecs, for 69 GCs in NGC 5128 measured with both our IMACS study and HST/ACS (McLaughlin et al. 2007). The solid line is a 1:1 relationship. A least squares fit (dashed line) gives $\mathrm{r}_{e}$ (IMACS) $=(1.02 \pm$ $0.04) \mathrm{r}_{e}(\mathrm{ACS})-(0.22 \pm 0.18)$. The IMACS uncertainties correspond to the standard deviation of the effective radii in the ISHAPE fitting, using three different concentration parameters $c=15,30,100$. The agreement between these independent techniques and datasets is evident. 


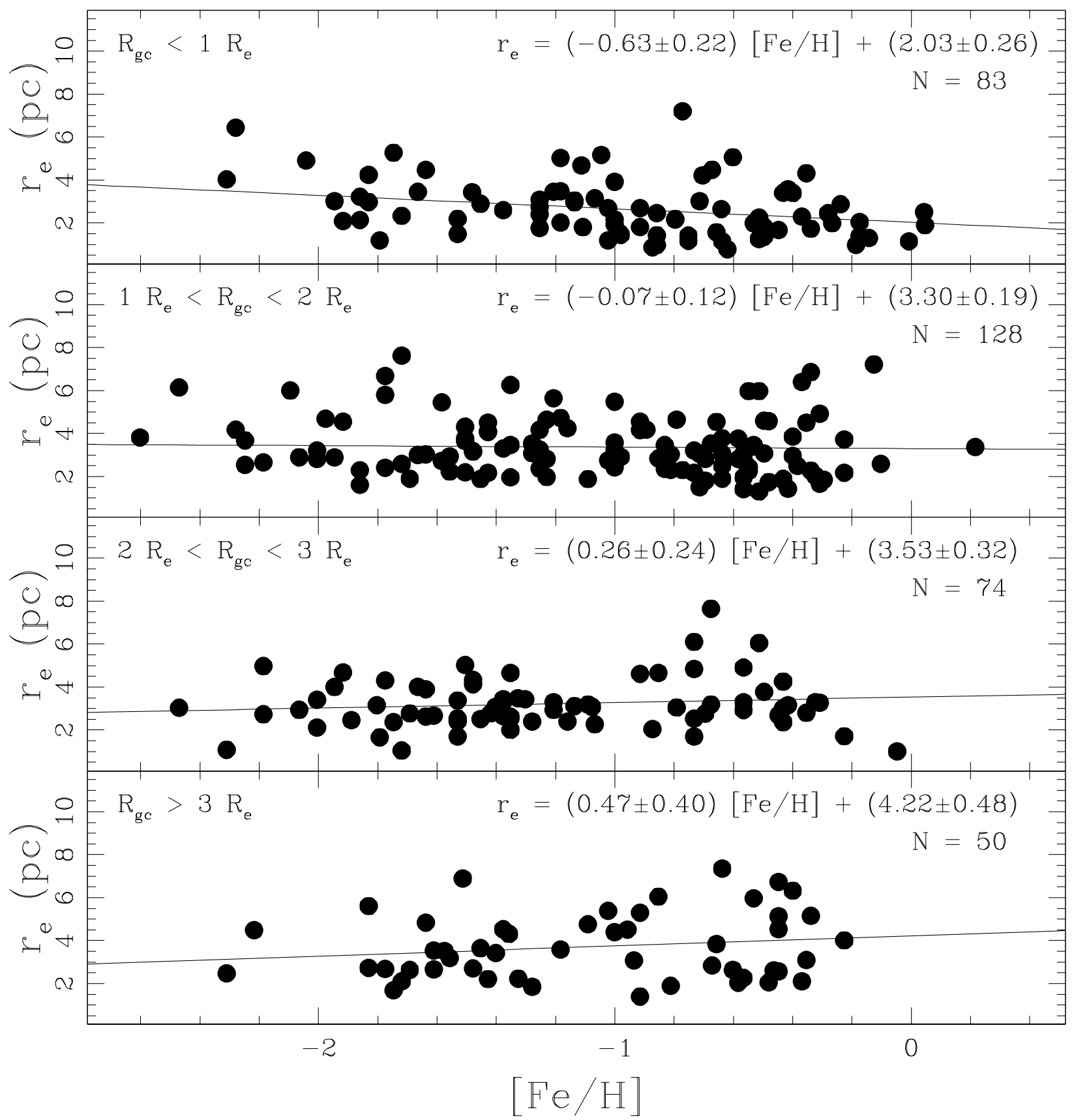

Fig. 2.- The effective radius, $\mathrm{r}_{e}$, as a function of $[\mathrm{Fe} / \mathrm{H}]$ for (from top to bottom) $\mathrm{R}_{g c}<1$ $\mathrm{R}_{e f f}$ of NGC 5128, $1 \mathrm{R}_{e f f}<\mathrm{R}_{g c}<2 \mathrm{R}_{e f f}, 2 R_{e f f}<\mathrm{R}_{g c}<3 \mathrm{R}_{e f f}$, and $\mathrm{R}_{g c}>3 \mathrm{R}_{e f f}$, where 1 $\mathrm{R}_{e f f}=5.1^{\prime}$. Least squares best fit and the number of GCs are indicated in each of the four panels. Only GCs with ellipticity less than 0.4 and $\mathrm{r}_{e}<8 \mathrm{pc}$ were considered in the fit. The change in the slope of the fitted lines is due primarily to an increase in the median size of red clusters. 


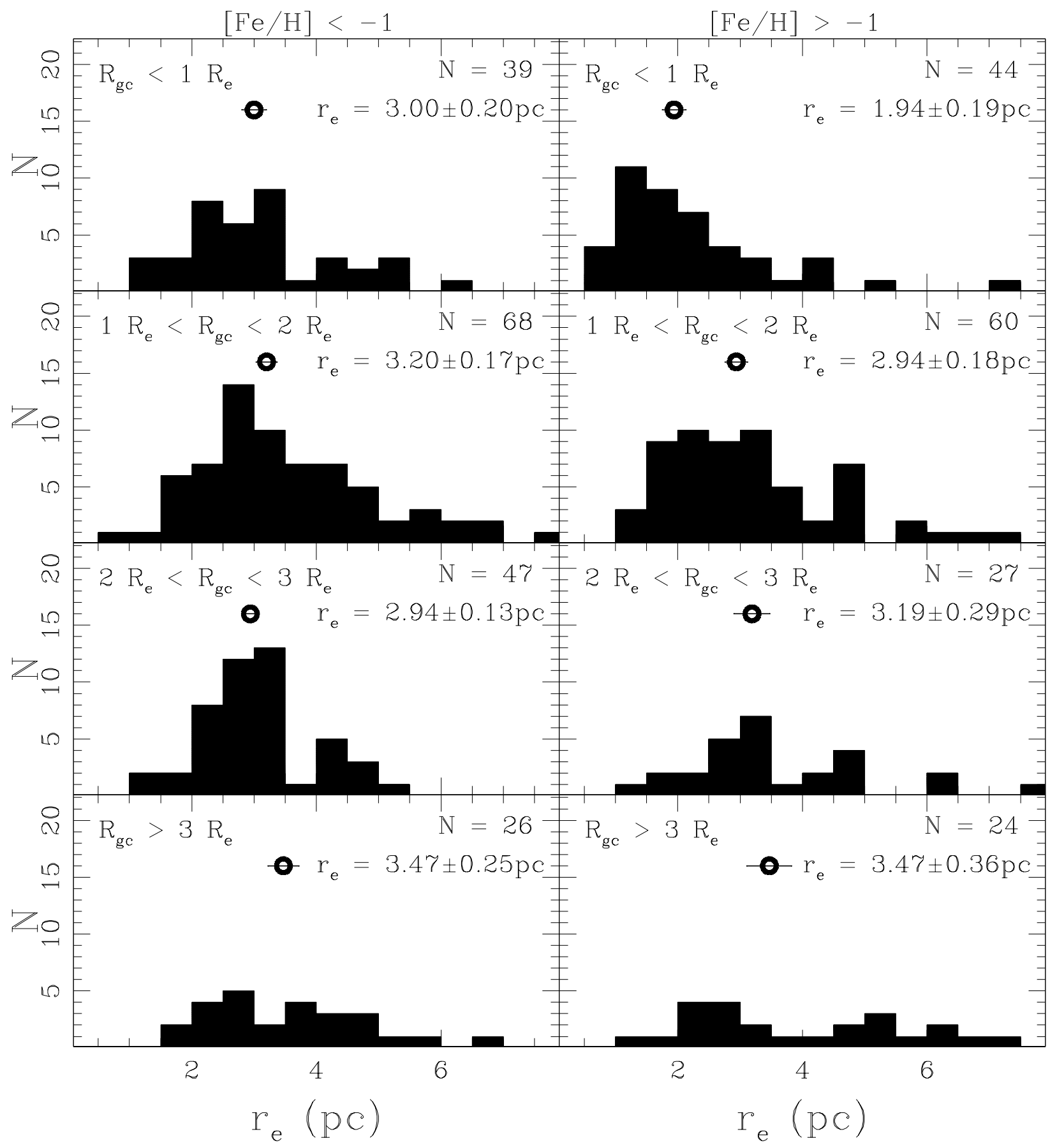

Fig. 3.- The distributions in $\mathrm{r}_{e}$ of the GCs in the same galactic effective radial bins as in Fig. 2, for metal-poor $([\mathrm{Fe} / \mathrm{H}]<-1)$ on the left and metal-rich $([\mathrm{Fe} / \mathrm{H}]>-1)$ clusters on the right. Associated number of GCs and the mean $\mathrm{r}_{e}$ (open circle) are labelled in each bin. Outside $3 \mathrm{R}_{\text {eff }}$ of the galaxy, the data on the GC population suffers from incompleteness and spatial bias. The open circle in each distribution gives the median value for the $r_{e}$ and its formal error for each subsample. Metal-poor clusters show no significant change of $r_{e}$ with $\mathrm{R}_{g c}$, while, on average, metal-rich clusters follow a clear trend for larger $r_{e}$ with $\mathrm{R}_{g c}$. 


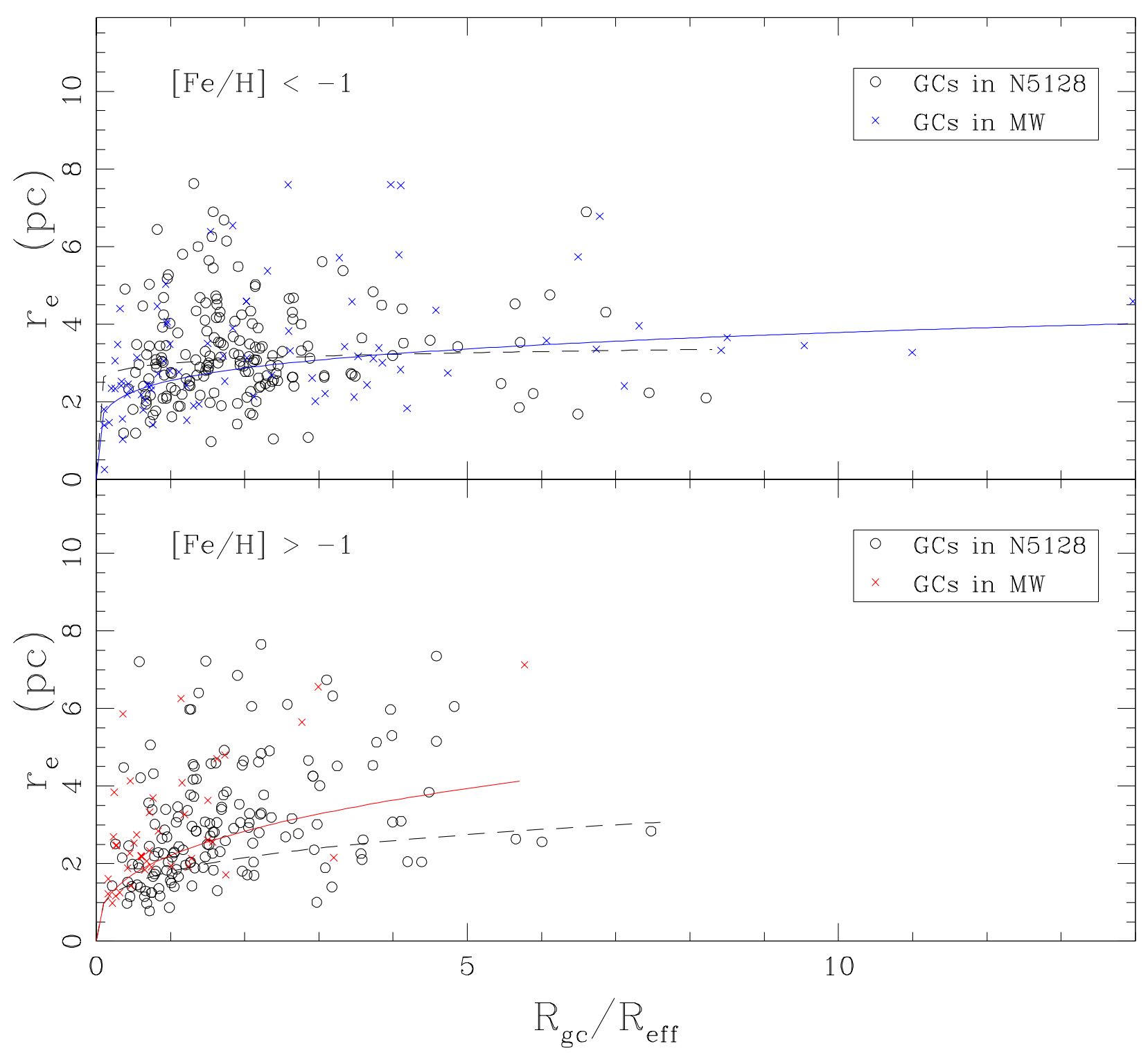

Fig. 4.- The effective radius, $\mathrm{r}_{e}$ in pc, as a function of projected galactocentric radius, $\mathrm{R}_{g c}$ in kpc, for the GCs in NGC 5128 (open circles) and in the Milky Way galaxy (crosses) for both the metal-poor GCs $([\mathrm{Fe} / \mathrm{H}]<-1)$ on the top and metal-rich GCs $([\mathrm{Fe} / \mathrm{H}]>-1)$ on the bottom. The Milky Way GC data is taken from Harris (1996) with the projected galactocentric radius defined as $\mathrm{R}_{g c}=\sqrt{y^{2}+z^{2}}$ and $\mathrm{R}_{e f f}=2.7 \mathrm{kpc}$ (de Vaucouleurs \& Pence 1978). Best fit curves of the form $\mathrm{r}_{e}=\mathrm{c}\left(\mathrm{R}_{g c} / \mathrm{R}_{e f f}\right)^{\alpha}$ yield $(\mathrm{c}=3.22 \pm 0.12, \alpha=0.05 \pm 0.05)$ and $(\mathrm{c}=2.76 \pm 0.14, \alpha=0.26 \pm 0.06)$ for the metal-poor and metal-rich GCs in NGC 5128 (dashed curves) along with $(\mathrm{c}=3.02 \pm 0.17, \alpha=0.17 \pm 0.04)$ and $(\mathrm{c}=3.16 \pm 0.21, \alpha=0.36 \pm 0.07)$ for the metal-poor and metal-rich GCs in the Milky Way (solid curves). 